72 Tropelías. Revista de Teoría de la Literatura y Literatura Comparada, 32 (2019) Javier Domingo Martín

\title{
MONSTRUOS INFERNALES EN MIS SÍLABAS. ECOS FANTÁSTICOS Y MARAVILLOSOS EN LA POESÍA DE CARLOS EDMUNDO DE ORY
}

\author{
MONSTRUOS INFERNALES EN MIS SÍLABAS. WONDERFUL AND \\ FANTASTIC ELEMENTS IN CARLOS EDMUNDO DE ORY'S POETRY
}

\author{
Javier DOMINGO MARTÍN ${ }^{1}$ \\ Universidad Complutense de Madrid \\ domingomartinjavier@gmail.com
}

\begin{abstract}
Resumen: En este artículo, analizaremos la poesía fantástica de Carlos Edmundo Ory a partir del estudio de los elementos maravillosos y fantásticos que en ella aparecen y de los poemas que pertenecen propiamente al género. Concluiremos que lo fantástico en Ory es una herramienta epistemológica que le permite explorar áreas de lo real inaccesibles al conocimiento racional, así como un espacio de reflexión sobre la escritura poética y la identidad personal, que es, signo de la posmodernidad, inestable y problemática.
\end{abstract}

Palabras clave: Carlos Edmundo de Ory; Postismo; Poesía fantástica; Posguerra.

Abstract: In this essay, we will study Carlos Edmundo de Ory's poetry by anylising the presence of marvelous and fantastic elements and by identifying the poems which properly belong to this subgenre. We will conclude that the fantastic is an epistemological tool which allows Ory to explore irrationality and to debate poetic writing and postmodern subjective identity in its unstable and problematic form.

Keywords: Carlos Edmundo de Ory; Postismo; Fantastic Poetry; Spanish Postwar.

\footnotetext{
${ }^{1}$ La realización de este trabajo se inscribe dentro del marco de los contratos predoctorales para la Formación de Profesorado Universitario (FPU) que financia el Ministerio de Educación, Cultura y Deporte. Número de referencia: 2016/03916.
} 


\section{Introducción}

Carlos Edmundo de Ory (1923-2010) es uno de los más radicales poetas de la posguerra española. Su obra, dividida en los tres jalones programáticos que marcaron su rumbo como poeta y agente cultural —Postismo (1945), Introrrealismo (1951) y Atelier de Poésie Ouverte (1968) - , fue una propuesta singular y originalísima en un ambiente cultural de marcado carácter realista (neoclasicismo garcilasista, neorromanticismo y poesía social). En este trabajo, propondremos una lectura del poeta desde el punto de vista de la incidencia de lo fantástico y lo maravilloso en sus versos. De un modo paralelo a su producción narrativa —que se ha publicado recientemente en la editorial Cátedra bajo el título de Cuentos sin hadas (2017)_-, su poesía ${ }^{2}$ refleja un interés insólito en su época por lo fantástico.

Como todo aquel que se enfrenta a la poesía de corte fantástico, nos encontramos con un obstáculo inicial: la afirmación tajante del teórico clásico sobre lo fantástico, Tzvetan Todorov, según la cual el género poético es incompatible con lo fantástico:

$\mathrm{Si}$, al leer un texto, se rechaza toda representación y se considera cada frase como una pura combinación semántica, lo fantástico no podrá aparecer: exige, como se recordará, una reacción frente a los acontecimientos tal como se producen en el mundo evocado. Por esta razón, lo fantástico solo puede subsistir en la ficción; la poesía no puede ser fantástica (aunque existan antología de «poesía fantástica...»). En una palabra, lo fantástico implica la ficción (Todorov, 1980: 50).

No obstante, en los últimos años algunos investigadores interesados en estudiar la presencia de lo fantástico en la poesía hispánica contemporánea se han enfrentado, con mayor o menor detenimiento, a las tesis de Todorov. Así, por ejemplo, Xaime Martínez, en un artículo sobre la poesía española de ciencia ficción, se hace eco de ello y señala, en primer lugar, la necesidad de distinguir la poesía narrativa (mucho menos problemática) y la lírica, en la que también observa «el conflicto entre realidad e irrealidad que está en el centro de las literaturas del extrañamiento» (2018: 384). También Luis Vicente Aguinaga defiende la presencia de lo fantástico para la lírica, asumiendo que, aunque en menor medida que en la novela, esta presenta elementos ficcionales y narrativos:

La poesía lírica, de contar con elementos narrativos o incluso ficcionales, cuenta con ellos de otro modo, no con arreglo a los procedimientos de la novela o del cuento. Luego, que lo fantástico implique a la ficción de ninguna manera justifica la exclusión de la poesía lírica: mientras haya componentes narrativos o de ficción en esta última, sin duda pervertidos y anómalos con respecto al canon de la novela y el cuento, podrá ocurrir en ella la irrupción de lo fantástico (2014).

\footnotetext{
${ }^{2}$ Es una lástima no contar todavía con la edición de las obras completas de uno de los poetas españoles recientes de mayor interés. Su poesía, dispersa en revistas y periódicos, se ha recogido en sucesivas antologías que dan una visión, aunque parcial, bastante completa del conjunto de la obra de Ory. Para este trabajo, pues, recurriremos a las más extensas (1974, 1978, 1990), así como a libros particulares especialmente interesantes para este trabajo: Los poemas de 1944 (1973), Lee sin temor (1976) y Soneto vivo (1988).
} 
Es Dolores Phillipps-López, sin embargo, quien se ha ocupado de este asunto con más detalle. Reelaborando las teorías del teórico francés Michel Viegnes, defiende la coexistencia en el poema de un plano literal, figurado, referencial, junto al metafórico y alegórico: «la persistencia posible de lo fantástico en un alegorismo o "simbolismo sutil" que mantuviera una superposición de los dos niveles de sentido figurado y literal, creando como un halo sugestivo alrededor de los hechos, sin alterar su poder de estupefacción» (2015: 140-141). De este modo, la organización del discurso poético «no indica de por sí cancelación de la facultad de denotar», y mantiene, en consecuencia, su capacidad mimética: «se trataría de una reinterpretación o — como la llama Ricoeur — una redescripción del universo referencial, que no anula el universo referencial» (2015: 141).

Aceptado este presupuesto, habremos de tener en cuenta los diferentes conceptos que delimitan nuestro objeto de estudio, y que ha estudiado David Roas: lo fantástico, lo maravilloso en sus diferentes variantes (lo real maravilloso y lo maravilloso cristiano) y lo neofantástico (2001). Para este trabajo nos interesa muy especialmente su concepción de lo fantástico en cuanto herramienta de conocimiento de una realidad inestable y compleja:

El relato fantástico pone al lector frente a lo sobrenatural, pero no como evasión, sino muy al contrario, para interrogarlo y hacerle perder la seguridad frente al mundo real [...] Basado, por tanto, en la confrontación de lo sobrenatural y lo real dentro de un mundo ordenado y estable como pretende ser el nuestro, el relato fantástico provoca - y, por tanto, refleja - la incertidumbre en la percepción de la realidad y del propio yo (2001: 8-9).

La conclusión es que «la literatura fantástica pone de manifiesto las problemáticas relaciones que se establecen entre el lenguaje y la realidad, puesto que trata de representar lo imposible, es decir, de ir más allá del lenguaje para trascender la realidad admitida» (2001: 30), aspecto fundamental para entender la relación de Ory con el género.

Alazraki, por su parte, se ha ocupado de estudiar la literatura neofantástica, que Roas denomina fantástico contemporáneo. Se diferencia en tres puntos fundamentales: (1) la visión: «lo neofantástico asume el mundo real como una máscara, como un tapujo que oculta una segunda realidad que es el verdadero destinatario de la narración neofantástica» (2001: 276); (2) la intención: son metáforas epistemológicas «que buscan expresar atisbos, entrevisiones o intersticios de sinrazón que escapan o se resisten al lenguaje de la comunicación» (2001: 277); (3) la mecánica: «el relato neofantástico prescinde también de los bastidores y utilería que contribuyen a la atmósfera o pathos necesaria para esa rajadura final. Desde las primeras frases del relato, el cuento neofantástico nos introduce, a boca de jarro, al elemento fantástico» (2001: 279).

La poesía de Ory presenta ecos de lo maravilloso, e incluso elabora una teoría poética en esta misma línea: el poeta como mago, la palabra en relación con lo sagrado. Pero también de lo fantástico en términos estrictos: la aparición de lo ominoso en sus versos, alejados del realismo al uso de la época, funciona como una herramienta cuestionadora de lo real, en su dimensión epistemológica y ontológica, así como de la propia noción del individuo. 


\section{La propuesta del postismo en el contexto poético de posguerra}

La obra poética de Ory está hondamente marcada por su adscripción inicial al postismo, uno de los grupos fundamentales de la poesía española de los años 40-50, fundado por Eduardo Chicharro, Silvano Sernesi y el propio Ory (a estos se sumarían otros con el tiempo: Gabino Alejandro Carriedo o Ángel Crespo, por ejemplo). El grupo, explicitado como tal en los tres manifiestos publicados en Postismo (1945), La Estafeta Literaria (1946) y El Minuto (1947), más un cuarto inédito (fechado en 1947 o 1948), se señaló como un post-ismo, el movimiento que va después de todos los ismos (Pont, 1987: 254).

Se sitúan de lleno, pues, dentro de lo que Adorno considera arte negativo: «en las dificultades en que los llamados ismos se enredan, se expresan las dificultades de un arte emancipado de su obviedad» (2004: 41). También cumple la máxima adorniana de un arte que es la negación de lo real, su cuestionamiento: figura en el primer manifiesto postista la intención de trascender un arte basado en la representación realista del mundo: «[si] nos atenemos a la reproducción pedestre de un trozo de lo que tenemos ante los ojos, no haremos sino un pobre facsímil de lo pobremente visto» (en Pont, 1987: 251).

La crítica ha señalado «una profunda incompatibilidad entre el postismo y las estéticas más sobresalientes de la época» (Navas Ocaña, 2000: 18), que en sus diferentes variantes se pueden agrupar por su común carácter realista y su vuelta al impulso mimético previo a las vanguardias históricas. No se nos escapa, pues, el efecto de oposición hacia la abundante poesía amorosa contemporánea de un texto como «Trabajo de amor» (Ory, 1990: 131-132), un ejercicio de animalización de la mujer en un contexto erótico: «Cuando tu bello hocico beso y muerdo [...] / oigo rodar tu hipo tranquilo y plateado [...] / y sonó tu salada voz de harpía [...] / para de noche amar tus ojos de ave / te encuentro y te trabajo». La poesía de Ory, pues, desvaloriza (Genette, 1989: 444) figuras claves de la tradición, como Rubén Darío en «Superestructuras»: «Otro ejemplo del mismo Rubio Diario / ¡Ya viene el cortejo! / Ya viene el cartero / digo yo» (Ory, 1990: 300); Jorge Guillén en «Cántico» (Ory, 1990: 193), al que no menciona pero con el que se relaciona mediante el título y la imitación de algunos usos propios del vallisoletano: «y todo el jardín es himno», «algo en los lejanos aires», aunque en este texto el canto del «mundo está bien hecho» se ha transformado en «las rosas vacías / [que] llenan de huesos la noche»; o el T. S. Eliot de The Waste Land, que es un hipotexto explícito («Aún se mezclan aquí los versos de Eliot») de «A la memoria de un ex cadáver» (Ory, 1990: 224-225), cuya búsqueda angustiosa del agua como renovación de la vida queda reducida al imperativo: «Anda ve y siéntate y bebe un vaso de agua».

Esta disociación entre el lenguaje y realidad que señalaba Roas en el empeño de representar lo imposible fantástico se convierte, en la poética de Ory, en voluntad explícita de problematizar la realidad mediante la creación de un lenguaje propio, cuestionar nuestra seguridad sobre la misma mediante un extrañamiento radical. Habla, pues, del poeta como aquel que «a la realidad le pone guantes» (Ory, 1990: 144), aquel que, como afirma Granell Trías, «opera por disolución en un mundo que no puede ser ya el mundo real» (2001: 251); poseedor de una lengua exclusiva — «óyeme con mi 
idioma de poema» (Ory, 1990: 269)—, que inventa el lenguaje al mismo tiempo que se escribe: «La nunca metáfora riendo en mi lengua / La nunca metáfora sin inventar aún» (1990: 273). Así lo manifestaba, de hecho, en una poética de Lee sin temor: «Ponte un vestido que no existe / Siéntate en una silla que no existe / Lee un libro que no existe / Besa a una mujer que no existe» (1976: 68). Y esta voluntad se convierte en imperativo en un texto como «Poeta joven» (1990: 206): «Pero la vida no importa importan las manos / No llaméis a las cosas coged las cosas / Y si arden enfriadlas / enfriadlas».

Algunos poemas, por otro lado, plagados de imágenes creacionistas — «Como un niño con mocos / me caen del alma cítaras» (1990: 145) — , insisten en el punto de vista particular del poeta: «Mi poesía no sale por la puerta de todos / sale por la rendija del mundo» (1990: 145). Y se subraya la frágil dicotomía en que se mueve el escritor, entre lo estrictamente artístico, «un hombro mío soporta un alfiler», y lo referencial, «el otro hombro puso un anuncio en el periódico». Esta misma tensión se manifiesta de manera más compleja en «Lección de apertura a los cursos de mitologías orientales en la Facultad de Letras de la Universidad de X». En él, con un comienzo convencional que remeda los discursos parodiados en el título, se tuerce al extender los saludos iniciales, «Señor Rector / Señor Decano de la Facultad de Letras» (1990: 302), hasta aquello hacia lo que solo el poeta centra su atención: «Niños / criadas de servir / trabajadores obreros / gatos y perros / hormigas».

Vemos, pues, cómo dentro de la poética general del postismo y de Ory en concreto alienta un propósito epistemológico de carácter similar al de la mejor literatura fantástica. Así lo señala el propio poeta en un prólogo al frente de su volumen antológico Energeia: «Sin llegar a convertirse en brujos, ciertos poetas de nuestro Occidente racional odiaron suficientemente la descripción del mundo apañada por la conciencia adquirida, en el reino hominal, dado por objetivo y revelado científicamente por el conocimiento fáustico» (1978: 15). Lo fantástico, pues, como una herramienta epistemológica que permitirá explorar aquellas zonas de la realidad que están vetadas para el conocimiento racional. La relación de Ory con el género, además de esta conexión de carácter general, se extiende a un número considerable de referencias a temas y motivos de dicho universo, de los que ahora hablaremos.

\section{Ecos fantásticos y maravillosos en la poesía de Carlos Edmundo de Ory}

Ferrer Solá ha estudiado el universo maravilloso de otro autor de la vanguardia de posguerra: Miguel Labordeta. Analiza en su trabajo su «retórica de magos, brujos, herejes, visionarios, malditos y heterodoxos» (1999: 333) y concluye que, además de ser una forma de ostentar «una desarraigada procedencia cultural» (1999: 333), se convierte en «una esencial indagación sobre ese estado indefinible del ser» (1999: 339) ${ }^{3}$. Del mismo modo, la poesía de Ory está plagada de este tipo de referencias: ogros: «Montado en un caballo que no tiene cabeza / Hombre rubio del Norte tú no puedes / saber nada del ogro sangriento que yo soy» (1973: 18), «Como la ogresa antigua / tu reclamo supone

\footnotetext{
${ }^{3}$ La poesía fantástica española es un campo de investigación todavía por explorar, con las notables excepciones del trabajo citado de Ferrer Solá y algunas menciones aisladas en estudios sobre otros géneros. Aunque se centra fundamentalmente en la poesía de ciencia fícción, es de rigor señalar también el estudio de Xaime Martínez (2018).
} 
/ satisfacción autónoma» (1976: 36); brujas y brujos: «El brujo del reloj / en su carro de horas con las ruedas de seda (1973: 83), «Oh galope oh viento de brujos» (1976: 25); o dragones: «Y no viste al dragón sino solo señuelos / Majestad mariposa debajo de la cúpula» (1976: 97), así como ambientes goyescos de aquelarre: «vi al diablo quemar jazmines una vez / Los muertos por las noches tocan flautas» (1973: 18).

Hay que relacionar la presencia de esta imaginería maravillosa con la doble concepción del poeta de su labor de escritor como magia, por un lado, y como asunción del mal contemporáneo y de lo demoniaco, por el otro. Jaume Pont, de hecho, describía la poesía de Ory «como magia o poder transmutatorio de las realidades exteriores en interiores» (1987: 252), y el propio autor lo manifestó así en múltiples textos: «maníaco admirable y aplicado / El trabajo (magia) / de escribir / Escribir a mandíbula batiente / La magia (trabajo) / del poeta» (1976: 21); «Mi arte de magia en un mundo sin magia / es el arte de la nostalgia del origen» (1990: 258). De ahí que su poesía adquiera muchas veces tintes de sortilegio fonético: «Entre lejos allí / Lo lejos que se aleja lejándose / El lejanía lejanosidad / Tu lejano lejar de alejadez» (1976: 125).

Asimismo, lo maravilloso le permite adoptar un imaginario del mal en su poesía. No son pocos los poemas en que, metapoéticamente, se manifiesta este trabajo sobre lo negativo del mundo: «Me arrastro sobre la tierra como un montón de estiércol» (1990: 217), «Tu boca pura pon en el abismo» (1990: 235), «Soy lluvia de belleza / que cae gota a gota en el abismo» (1990: 291), o en «Poderes» (1990: 153): «Sobre todo yo canto yo unifico la noche / Yo la nombro y repito su gran señal de duelo / Me va pesando el alma y me va dando alma / el triste triste nombre que encierran mis poderes». La perspectiva, sin embargo, no es trágica, sino parodia de lo trágico. Así lo apunta Ory: «Lo mismo soy sublime que grosero / dramaturgo del llanto y de la risa» (1990: 248) o «Pero mi estado verdadero es / un estado de payaso natural» (1990: 147). Y se hace patente en textos como «En un café», a medias entre lo risible y lo trágico: «He vuelto ahora sin saber por qué / a estar triste más triste que un tintero» (1990: 185).

En otras ocasiones, el poema no se limita a reflejar lo negativo sino que lo asume como propio, en el modelo de Baudelaire estudiado por Adorno: «Lo nuevo es hermano de la muerte. Lo que en Baudelaire se da aires de satanismo es la identificación con la negatividad real de la situación social, que se refleja a sí misma como negativa [...] El arte es moderno a través de la mímesis de lo endurecido y alienado; habla de este modo, no renegando de lo mudo» (2004: 36). Es claro, por ejemplo, «Pferdefuss» (Ory, 1990: 210): apología del pecado — «Cuéntame todo eso porque amo tus pecados / y tu asquerosa alma me fascina»— en un ambiente mortuorio: «Todavía podemos besarnos en la gruta / de la desgracia nuestra con besos de ladrillo». En la poética «Lírica absoluta», que describe, por otra parte, un ambiente de novela gótica — «He aquí que moramos en la ciudad tenebrosa / en donde enmudeció la época noble de los monjes / y el ideal lírico de la oscuridad» (1976: 98) — se disculpa por su estética de lo monstruoso: «No me calumnien a mí por mi oscuridad / viendo monstruos infernales en mis sílabas / de locura y de radium monástico» (1976: 98). O «Las palabras», en que 
vincula directamente la labor del poeta con lo infernal: «[las palabras] Llegaban solas traídas por demonios / y brotaban de pozos de cisternas / casi infernales o de vagos lagos» (Ory, 1990: 158-159).

Además de estos poemas, encuadrados en la órbita de lo maravilloso, la obra de Ory presenta un corpus nada desdeñable de textos que se sitúan con pleno derecho dentro del ámbito de lo fantástico. Un primer grupo es aquel en que, en un ambiente cotidiano, irrumpe lo fantástico — lo ominoso- a través de la visión del poeta. Es el caso de dos poemas de 1944 que se citan mutuamente. El primero de ellos comienza así: «He comenzado a ver cosas / ramas muertes mujeres [...] / Qué extraño es ver de pronto / cosas que no se vuelven a ver / misteriosos caballos solitarios / como sin dueños» (1973: 46). La incomprensión del poeta se traslada al lector cuando, en este contexto de visiones del más allá, el protagonista del poeta reclama diferentes elementos cotidianos: «iMadre yo quiero un pantalón / bien planchado! [...] / ¡Quiero tener un peine mío!» (1973: 46). Otro poema de este primer conjunto comienza también remarcando la cotidianeidad: «Solo otra vez aquí en mi cuarto veo / mi toalla mis libros las sillas ocupadas / Las maletas vacías por el suelo» (1973: 47). Es entonces cuando se produce la visión, que incluye una cita del poema anterior: «Y yo en la cama triste / escribiendo o rezando y viendo cosas / "ramas mujeres muertes"» (1973: 47). De este proceso no hay otra conclusión que la incomprensión: «No comprendo por qué Yo no comprendo / nada Yo no comprendo nada» (1973: 47).

Esa tríada de elementos que autocita Ory - «ramas mujeres muertes»— tendrá gran importancia en su poesía fantástica. En primer lugar, la figura de la mujer fantasma que va a aparecer una y otra vez en sus versos, siempre como un misterio indescifrable: «En la tarde con lluvia una mujer sin sexo / una mujer de aire y gasa yerra Voy / hacia ella y no existe Veo solo sus ojos / y su boca y su pelo Pero no existe no» (1973: 83); «Aparece la mujer en mi vida / Aparece y desaparece / como un fuego fatuo o un peligro» (1990: 271). Las ramas y las muertes, por otro lado, forman parte de una serie de poemas que reflejan el miedo hacia el exterior y al levantamiento de los muertos. En «Más muerte», por ejemplo, afirma: «Voces frías heladas de muertos que se quejan / a través del verdín como campos de odio / Y si lloran los muertos que lloren mientras yo / me pregunto si acaso ya me quedan preguntas» (1973: 58). «Exterius» es un largo poema que plantea el temor del poeta ante los muertos que acechan al exterior: «Ven aquí sobre mis rodillas vamos a ver / lo que pasa en el bosque [...] Sábelo los muertos matan a los vivos» (1976: 104). El texto toma la forma de un monólogo dirigido a un interlocutor del que no sabemos nada y que es continuamente interrumpido por referencias a la amenaza exterior: «¿Quién está ahí ¿Qué luz es esa? ¿A qué vienes tú? [...] / ¿Quién viene? ¿Quién me llama? Nadie...» (1976: 104-105). Esta inconcreta amenaza se hace explícita en la estrofa siguiente: «iSi vierais cómo baja el barómetro! / Ya lo barruntaba / Se ha levantado el viento Ya lo barruntaba / Los espectros arman tumultos / Los muertos surgen Los vivos sueñan» (1976: 105). En todos estos casos la irrupción de lo fantástico, pues, subraya los difusos límites que separan la vida y la muerte, y plagan su poesía de presencias del más allá que no hacen sino señalar la condición efímera y problemática del sujeto contemporáneo.

A resaltar este precepto contribuye un grupo de poemas que están escritos desde la perspectiva del muerto: son, pues, monólogos en la órbita de lo neofantástico que asumen la normalidad del 
discurso post mortem como la única posibilidad de la palabra poética posmoderna, rebasados ya todos los límites. Es el caso de «Pferdefuss», otro diálogo del que solo tenemos las palabras de un interlocutor. En él, el sujeto le hace una serie de preguntas acerca de la vida pasada: «Y el tren en que viajábamos / ¿sigue dando pitidos a través de los pálidos / jardines del abismo? [...] / ¿Dónde está nuestra isla? ¿Dónde nuestras palmeras?»(1990: 210-211). En un contexto que hace patente la asunción del mal y del pecado, una constante de la poética de Ory, como decíamos — «Cuéntame todo eso porque amo tus pecados / y tu asquerosa alma me fascina» (1990: 210) — y en la que no se escatima ninguna referencia escatológica: $«_{i} A y$, yo tampoco puedo tampoco yo resisto / la triste cucaracha que se atasca en mi boca!» (1990: 211). Poema, en fin, donde el erotismo y la muerte, eros y tánatos, aparecen indisociablemente unidos desde su comienzo: «Quítate los zapatos y acaríciame / Estoy totalmente solo y pido caricias» (1990: 210), hasta sus últimos versos: «Todavía podemos besarnos en la gruta / de la desgracia nuestra con besos de ladrillo / La tapa de la fosa podemos todavía / levantar para que entre un poco de aire orondo» (1990: 211). Este mismo ánimo de mirar lo imposible desde la cotidianeidad se da, en fin, en un texto tan aséptico como este de Lee sin temor: «Escribir con una espada / Acariciar sin tener manos / Encontrar pedazos de luna en los bolsillos / Comprar una playa con gritos / Ir al infierno a ver a un amigo / Enviar una mano a su amada» (1976: 69).

El fantasma es otro elemento de gran recorrido en la poesía de Ory. Esta figura aparece como presencia de lo ominoso y desconocido que viene a turbar el espacio por excelencia de la cotidianeidad: la casa. Son numerosos, pues, los poemas que giran en torno a esa presencia misteriosa: «Sonata del jardín negro», por ejemplo, subraya el miedo del poeta ante «los bultos [que] siguen / con sus voces remotas»: «¿Quién danza? ¿Quién retira / brazos? ¿Quién boga ronca / debajo de los árboles? / ¡No gritad por las hojas! / ¡No moved por el agua / con los dedos sus ondas!» (1974: 17); o «Insondable insomnio», en el que se señala esta misma amenaza inconcreta: «Secos crujidos secas alas de silencio / Titila un ser en lo alto de la noche» (1978: 111). El ambiente nocturno, como es natural, es común a todos estos poemas y especialmente remarcable en los tres cuartetos de un poema sin título cuyos versos acaban, salvo el último, con la palabra «anoche». En su última estrofa aparece la presencia fantasmal: «Pero se han puesto negras las estrellas de anoche / Sigue chillando el pájaro que entró en el cuarto anoche / Ya juegan como anoche gimiendo como anoche / las sombras que parecen bichos en agonía» (1990: 303). Junto a este elemento, la ventana desempeña un papel fundamental, en cuanto espacio del entre que conecta el mundo de la cotidianeidad doméstica y el exterior ominoso. Esto es claro en un texto titulado significativamente «Poema que no pude proseguir por miedo» —recuérdese que Roas señala esta emoción como el efecto fundamental de lo fantástico (2001: 30)—, en el que además se juega con la confusión vigilia-sueño tan frecuente en estos poemas: «Vi en sueños algo que entraba sin cara / Estaba despierto en el sueño y me dormía / Estaba dormido en el sueño y desperté [...] / Me vio a mí una ventana / abierta y no me dijo / ciérrameciérrame» (1990: 245).

Dentro de este corpus es especialmente relevante el texto «Sobre unos fantasmas que asustaron a Ana», pues cumple a la perfección la máxima todoroviana de la vacilación: «de la duda entre una explicación natural y una explicación sobrenatural de los hechos narrados» (Roas, 2001: 15) de la que 
nace el efecto fantástico. El poema, como tantos otros, se construye como un diálogo del que solo conservamos las palabras del poeta, dirigidas a Ana —en «Insomnio», también un poema con fantasmas de por medio, encontramos la versión del diálogo completo: «Apaga la llama larga / — ¿Por qué se mueve esa estatua? / ¿Son de mármol los fantasmas? / _Hija!» (1990: 116)—. El poeta intenta tranquilizar a la mujer por unos fantasmas de cuya existencia no se duda en la primera estrofa: «Acaban de irse ponte te lo ruego / ponte las zapatillas loca Ana / Yo los he visto ir me han dicho adiós / y sus lenguas de fuego han apagado» (1990: 126). Según avanza el poema, sin embargo, otra explicación será la que prevalezca: Ana, a quien le dice «visionaria mía», «nadie vi como tú que viera tanto», ha confundido lo que ha visto: «Acomoda tus ojos a la noche [...] / ique ves fantasmas donde estrellas hay! [...] / Eran sí tus cabellos te asustaron» (1990: 127). El último verso, finalmente, vuelve a la explicación sobrenatural del comienzo: «Yo pensé que en verdad eran fantasmas» (1990: 127).

Son muchas las variantes en torno a esta figura. Señalamos aquí dos significativas, como muestra patente de la variedad que adquieren los motivos fantásticos en la poesía de Ory. Cabe la posibilidad, entonces, de que el propio poeta sea el fantasma en cuestión. En el poema anterior se sugiere bromeando esta posibilidad: «Ahora soy yo el que te habla no lo dudes / ¿crees que soy un fantasma yo también?» (1990: 127), y en otros como «Baladas a una muchacha» es algo totalmente patente: «Sobre el aire el fantasma de siempre yo seré / Te dije que vinieras conmigo al parque triste» (1978: 44). Se da también la situación, en ocasiones, de que sea el fantasma el sorprendido ante ciertas capacidades del poeta: «También rio el fantasma que vi anoche y rio / porque le pude ver lleno de asombro» (1973: 22).

La casa encantada, finalmente, es otro de los temas fantásticos en los que la poesía de Ory insiste a lo largo de varios poemas. El poeta la denomina «la casa muerta» en dos sonetos de 1947 y 1976 . En ambos se subraya el estado de abandono en que se encuentra dicho espacio: «No el viento pasa No la lluvia pasa / Ni aun nadie se le acerca porque evita / el miedo que le da la casa muerta» (1988: 136); «La verja está cerrada y el jardín / mojado tanto en lágrimas de invierno [...] / ¿Entramos? ¡Poco importan las arañas!» (1988: 31). También en los dos casos se concluye con la metamorfosis del poeta en la propia casa: «Yo soy aquella la lejana casa» (1988: 136); «Yo mismo era la casa estando sin / nadie en la noche de mi fuero interno» (1988: 31).

Esto entronca con uno de los aspectos más remarcables de la utilización oryana de lo fantástico: la constatación, siguiendo a Rimbaud, de que el yo es otro [je est un autre], una ruptura con el contrato subjetivo, «con la falsa conciencia de encontrarnos ante nosotros mismos» (Mora, 2016: 229). La poesía de Ory, que tiene como uno de sus centros de significación la problemática posmoderna de la identidad, recurre a lo fantástico como una de las formas de subrayar el ego escindido o múltiple del sujeto contemporáneo; algo, por otra parte, que no es ajeno al género, como señala Campra en lo referente al desdoblamiento del yo como uno de los métodos de anulación de la identidad (2001: 165). En Ory esto es especialmente significativo en textos de un marcado carácter fantástico. Así pues, el poeta se desdobla en numerosas ocasiones y habla consigo mismo: «Al bosque del ensueño se marchó muy temprano [...] / El hada misteriosa le cogía una mano [...] / gritándome ¿soy yo? y él te dijo ¡soy 
tú!» (1978: 39); o bien se adentra en sí mismo y, tras un ejercicio de introspección, se da cuenta de lo artificioso de su identidad: «Penetro en lo negro de mí mismo / Ventanas y puertas están cerradas» (1976: 152); «En mi alma esta noche / golpea una ventana / ¿Habrá un diablo esta noche en mi alma? / era un niño dormido / y hoy soy otro / niño que da gritos» (1978: 45). No extraña, en este sentido, que muchos de sus poemas fantásticos concluyan con la conversión del poeta en la amenaza fantástica: «Cerré los ojos con vergüenza y lo veía / metido en mis adentros y yo era el coco mío» (1978: 228); «Entro llamo ay ay ¡Señorito! ¡Ay! ¡Ay! / No puede ser así usted no se parece / ¡Aparición! ¿Quién soy? [...] / No te pongas así no te asustes de mí / ¡Ayaymiseñoritoustedyanoeselmismo» (1990: 137). De ahí también que la reflexión sobre el nombre propio sea constante, entroncándose así con una línea de poesía autoficticia propia de la posmodernidad (Scarano, 2014). Así, insiste con excesivo entusiasmo en el mismo: «Yo me llamo Carlos Carlos de Carlos» (1976: 57), lo niega: «No me llamo Carlos» (1976: 67), o le quita, por su inoperancia, toda su importancia, en un contexto, por otra parte, de apariciones fantasmagóricas: «Llamadme como sea sin nombre como sea [...] / En mi cráneo abandonado en el silencio / Un fantasma se ríe de mí Lo sé lo sé» (1973: 51).

\section{Conclusiones}

Encontramos en la obra de Carlos Edmundo de Ory, pues, una presencia constante del imaginario de lo maravilloso. Su poesía, plagada de ogros y de brujas, así como su poética de sortilegio mágico, se constituye en una forma de abrir la escritura a otras esferas de la realidad inaccesibles al conocimiento científico-racional, así como un modo implícito de singularizarse - y marginalizarseen un contexto literario de marcado carácter realista

Asimismo, lo fantástico en la obra de Ory, lejos de ser un elemento marginal y accesorio, se convierte en marco genérico esencial para muchos de sus textos, que entronca con algunos de los puntos fundamentales de su poética particular y de la propuesta postista en general. Lo fantástico sirve a su propósito de cuestionar la realidad, de generar un extrañamiento radical sobre esta. El arte de vanguardia, tal y como señala Adorno, no puede reflejar la realidad si no es mediante su negación «soporto la inconsciencia del gran asombro que / me hace callar como un espejo negro» (Ory, 1990: 151) - , por lo que lo fantástico en cuanto marco de referencia es clave en este aspecto. De ahí que una parte significativa de su poesía esté marcada por la amenaza de lo ominoso: el fantasma, el no-muerto, la presencia innombrable. Lo demoniaco, con lo que el poeta se identifica, desemboca finalmente en una reflexión sobre la escritura poética y sobre la identidad personal, que es, signo de la posmodernidad, inestable y problemática. 
82 Tropelías. Revista de Teoría de la Literatura y Literatura Comparada, 32 (2019)

Javier Domingo Martín

\section{Referencias bibliográficas}

AdORno, Th. W. (2004): Teoría estética. Madrid, Akal.

AguinagA, L. V. (2014): «¿Existe la poesía fantástica?», en Leer por escrito. Disponible en: https://luisvicentedeaguinaga.wordpress.com/2014/02/11/existe-la-poesia-fantastica/ [13-062019].

AlAZRAKI, J. (2001): «¿Qué es lo neofantástico?», en D. RoAS, ed., Teorías de lo fantástico. Madrid, Arco/Libros, pp. 265-282.

CAMPRA, R. (2001): «Lo fantástico: una isotopía de la transgresión», en D. RoAs, ed., Teorías de lo fantástico. Madrid, Arco/Libros, pp. 153-191.

FERRER SOLÁ, J. (1999): «Brujos y demiurgos en la poesía española de postguerra: el surrealismo de Miguel Labordeta», en J. PONT, ed., Brujas, demonios y fantasmas en la literatura fantástica hispánica. Lleida, Edicions Universitat de Lleida, pp. 331-339.

GenetTE, G. (1989): Palimpsestos: la literatura en segundo grado. Madrid, Taurus.

Granell Trías, E. (2001): «La no novia: los poemas-collages de Carlos Edmundo de Ory», en J. Pont y J. Fernández Palacios, eds., Carlos Edmundo de Ory: Textos críticos sobre su obra. Cádiz, Diputación de Cádiz, pp. 249-258.

MARTíneZ, X. (2018): «Poesía 1900-2015», en T. LóPEZ-PELlisA, ed., Historia de la ciencia ficción en la cultura española. Madrid/Frankfurt, Iberoamericana/Vervuert, pp. 381-412.

Mora, V. L. (2016): El sujeto boscoso. Tipologías subjetivas de la poesía española contemporánea entre el espejo y la notredad (1980-2015). Madrid/Frankfurt, Iberoamericana/ Vervuert.

NAVAs OCAÑA, M. I. (2000): El postismo. Cuenca, El Toro de Barro.

ORY, C. E. de (1973): Los poemas de 1944. Madrid, Aguirabay. (1974): Poesía abierta (1945-1973). Ed. Jaume Pont. Madrid, Ocnos.

_ (1976): Lee sin temor. Madrid, Editora Nacional. (1978): Energeia. Barcelona, Plaza \& Janés.

_ (1988): Soneto vivo. Sant Viçenç dels Horts, Biblioteca de autores andaluces. (1990): Metanoia. Ed. Rafael de Cózar. Madrid, Cátedra.

(2017): Cuentos sin hadas. Ed. José Manuel García Gil. Madrid, Cátedra.

PhILLIPPS-LÓPEZ, D. (2015): «Ecos góticos y fantásticos en la poesía hispanoamericana del modernismo», en M. KunZ y J. M. SARDiÑAS, eds., Paisajes góticos. De lo fantástico y sus alrededores (siglos XVIII-XXI).Villeurbanne, Orbis Tertius.

PONT, J. (1987): El postismo. Un movimiento estético-literario de vanguardia. Barcelona, Ediciones del Mall.

ROAS, D. (2001): «La amenaza de lo fantástico», en Teorías de lo fantástico. Madrid, Arco/Libros, pp. $8-44$.

SCARANO, L., ed. (2014): Vidas en verso. Autoficciones poéticas (estudio y antología). Santa Fe, Universidad Nacional del Litoral.

TOdOROv, T. (1980): Introducción a la literatura fantástica. México, Premia. 